\title{
Materials for Laser Propulsion: "liquid" polymers
}

\author{
T. Lippert ${ }^{\mathrm{a}, *}$, L. Urech ${ }^{\mathrm{a}}$, R. Fardel ${ }^{\mathrm{a}, \mathrm{b}}$, M. Nagel ${ }^{\mathrm{b}}$, C. R. Phipps ${ }^{\mathrm{c}}$, A. Wokaun ${ }^{\mathrm{a}}$ \\ ${ }^{a}$ Materials Group, General Energy Department, Paul Scherrer Institut, Switzerland \\ ${ }^{\mathrm{b}}$ Laboratory of Functional Polymers, Empa, Switzerland \\ ${ }^{c}$ Photonic Associates, Santa Fe, USA
}

\begin{abstract}
The application of energetic polymers has resulted in an increased thrust in micro laser plasma thrusters compared to standard polymers. In this study we tested a novel concept for micro laser plasma thrusters, i.e. the application of liquid polymeric fuels, by using polymer solutions of the energetic materials with different viscosity. Shadowgraphy experiments suggest that for higher viscosity solutions ablation without splashing is possible, indicating that liquids are applicable as fuels in laser plasma thrusters. First thrust measurements on a viscous polymer solution confirmed this by yielding a specific impulse similar to a solid material.
\end{abstract}

Keywords: Laser plasma thruster, energetic polymer, plasma imaging, shadowgraphy, liquid polymer

\section{INTRODUCTION}

Micro-satellites for space applications allow to perform new tasks at reduced cost compared to conventional satellites $(>$ $100 \mathrm{~kg}$ ). Due to their low mass, these satellites have special issues regarding the propulsion system. The main requirements are a high specific impulse and a precise control of the thrust intensity, which are not fulfilled by conventional rocket thrusters. Therefore, there is a need to design novel systems for space micro-propulsion. One suitable technology for this purpose is the laser plasma thruster, where the impulse is provided by the laser ablation of a target $^{[1-6]}$. An infrared diode laser is focused onto a transparent ribbon coated with an energetic material, which is ablated by the laser pulse. The so-created plasma provides the thrust to propel the satellite along one axis. After each pulse, the ribbon is moved forward like in an audio tape. Energetic polymers are ideal target materials, because of their high energy density and low thermal conductivity, yielding precise ablation. ${ }^{[7-10]}$ Among them, the glycidyl azide polymer (GAP) doped with an infrared absorber (carbon nanoparticles) is an excellent candidate for this purpose, with specific impulses up to $550 \mathrm{~s}$ and an efficiency of up to $360 \%{ }^{[11,12]}$. The main disadvantage of this design is the use of a tape as fuel dispenser, which limits the mass of fuel on board the satellite. The alternative is to use liquid fuels, stored in a tank, with a good energy density and dispensed according to the needs. A solution of polymer is pumped through a nozzle, where it forms the target. After laser ablation, the target droplet is renewed by pumping again. Obviously, the solution have to be liquid enough to be pumped easily. On the other hand, previous work has shown that the ablation of liquid targets yields very low specific impulse ${ }^{[13]}$, e.g. of $19.3 \mathrm{~s}$ for water doped with a carbon ink ${ }^{[14]}$. This is essentially due to the splashing of the liquid, which drives out the laser energy into droplet formation instead of high-velocity plasma acceleration. Moreover, splashing materials would contaminate the laser optics and compromise the reliability of the system. Therefore, it is essential to to find appropriate conditions under which the solution does not exhibit splashing if one want to use liquid as fuel for laser plasma thrusters. In the present work, we investigated four liquid solutions of GAP (including one without polymer) plus a solid GAP target by shadowgraphy to test if liquid polymer solutions are applicable and even superior in micro laser plasma thrusters. Shadowgraphy was used to test whether a fuel composition could be identified that would behave similar to solid GAP upon ablation, i.e. whether splashing can be avoided.

\section{RESULTS AND DISCUSSION}

\subsection{Experimental}

Four liquid solutions (including one of without GAP, shown in Scheme 1 below) plus a solid target were investigated by shadowgraphy to test whether "liquid" polymer solutions are applicable and even superior in micro laser plasma

*thomas.lippert@psi.ch; phone ++41563104076; fax ++41563102688

High-Power Laser Ablation VII, edited by Claude R. Phipps

Proc. of SPIE Vol. 7005, 700512, (2008) · 0277-786X/08/\$18 - doi: 10.1117/12.782867

Proc. of SPIE Vol. $7005700512-1$ 
thrusters. Shadowgraphy was used to test whether a concentration could be identified that would behave similar to solid GAP, i.e. whether splashing - which would strongly reduce the performance of the polymer as fuel and contaminate the laser optics- can be avoided.

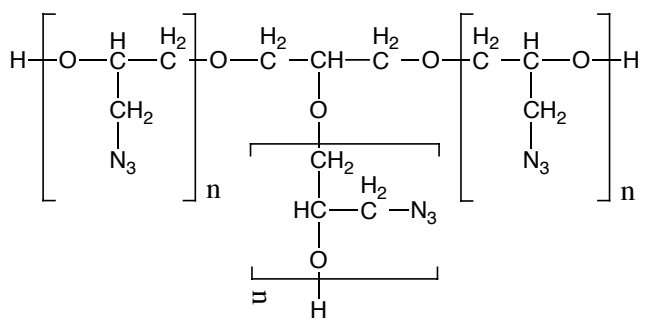

GAP polyol

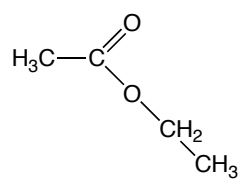

Ethylacetate

Scheme 1: Chemical structures of GAP and the applied solvent (ethylacetate)

As solvent ethyl acetate (EA, included in Scheme 1) was used, while for the absorber carbon nanoparticles (C) were applied. The following samples were prepared, which are summarized in Table 1 below)

Table 1: Composition of the samples analyzed by shadowgraphy.

\begin{tabular}{llll}
\hline Sample & GAP content $[\mathrm{wt} \%]$ & $\mathrm{C}$ content $[\mathrm{wt} \%]$ & EA content [wt $\%]$ \\
\hline Solvent & $0 \%$ & $1 \%$ & $99 \%$ \\
$28 \%$ GAP & $28 \%$ & $1 \%$ & $71 \%$ \\
$50 \%$ GAP & $50 \%$ & $1 \%$ & $49 \%$ \\
$70 \%$ GAP & $70 \%$ & $1 \%$ & $29 \%$ \\
Solid GAP & $99 \%$ & $1 \%$ & $0 \%$ \\
\hline
\end{tabular}

The liquid samples were placed on a PTFE slide and irradiated by $6 \mathrm{~ns}$ pulses at $1064 \mathrm{~nm}$ from a Nd:YAG laser (pump laser). The spot size was a circle of $300 \mu \mathrm{m}$ diameter. The illumination was obtained by a fluorescent dye pumped with $30 \mathrm{~ns}$ pulses at $308 \mathrm{~nm}$ from a $\mathrm{XeCl}$ excimer laser (probe laser). A camera with a zoom objective was used to image the process. The experimental setup is shown in Figure 1.

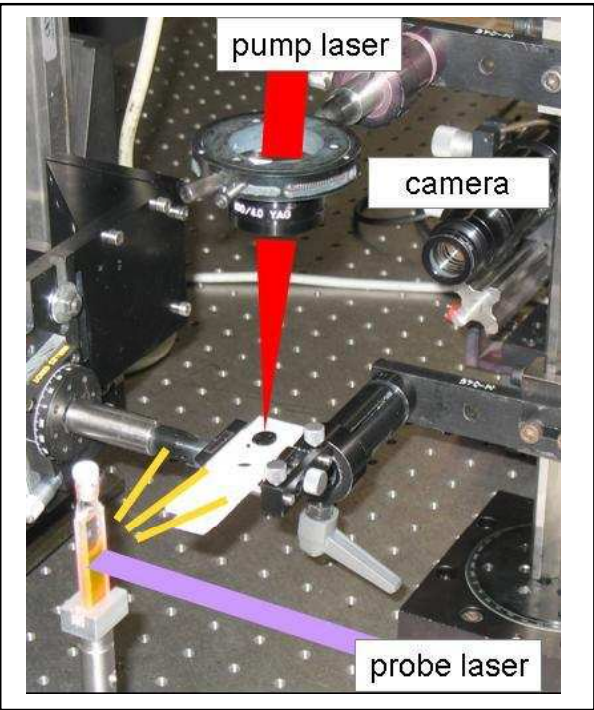

Figure 1: Overview of the experimental setup for shadowgraphy of liquid samples 


\subsection{Shadowgraphy}

Shadowgraphy was applied as experimental technique as it shows directly whether splashing is observed but also whether a solution behaves similar to solid GAP which is up to now the "best" solid fuel for laser micro plasma thruster ${ }^{[12]}$ with an efficiency of up to $360 \%$.

\subsubsection{Solid GAP}

Solid Gap has been analyzed to allow a direct comparison between the viscous polymers and the solid material. The ablation of solid GAP+C targets produces a shockwave (visible at 1 and $2 \mu$ s in Figure 2.) and particles are ejected (at higher fluences, i.e. used for "plasma" conditions, particles cannot be observed because the plasma is too bright).
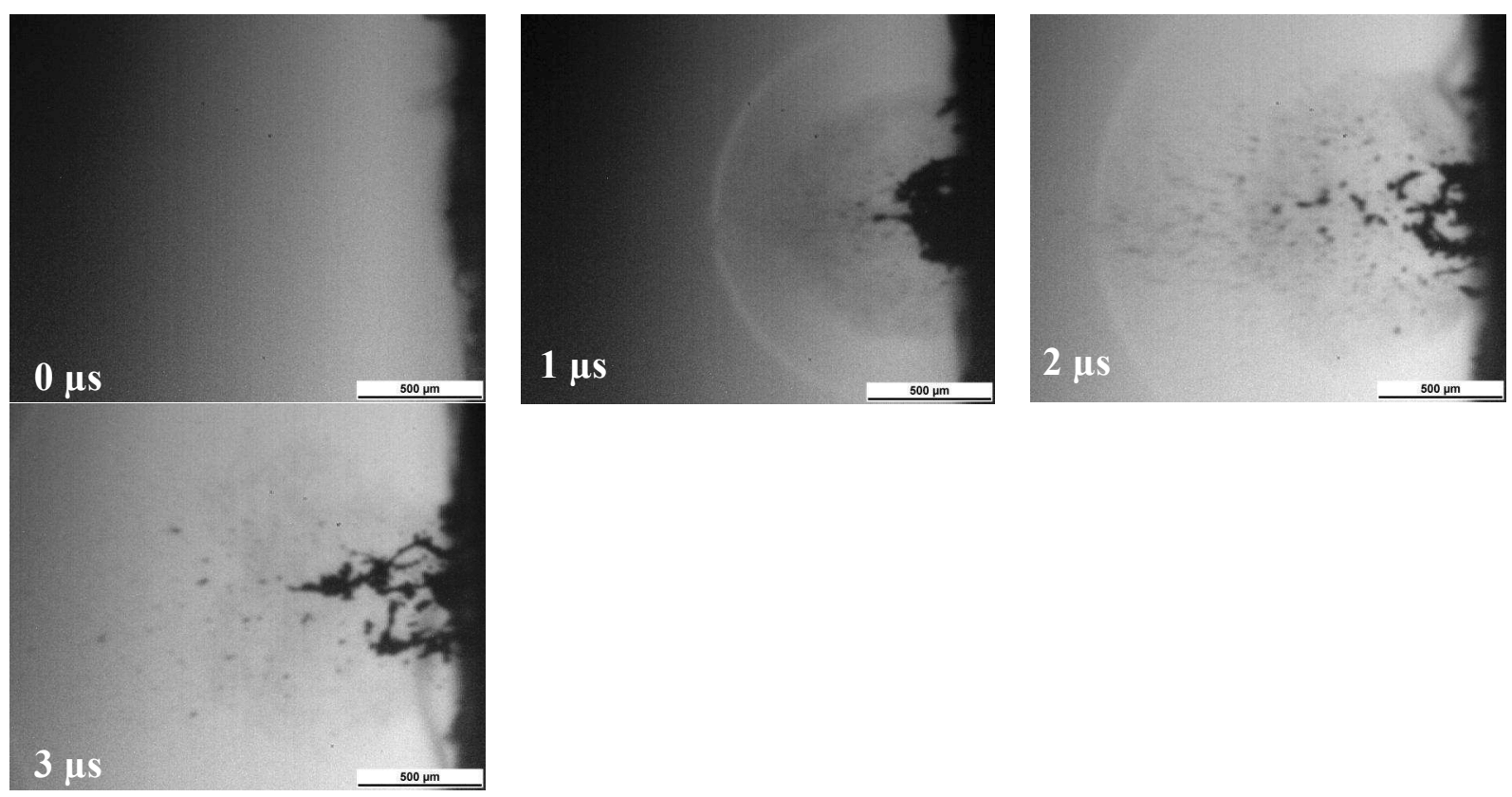

Figure 2: Sequence of pictures taken for solid GAP with $1 \% \mathrm{C}$ particles at $3.2 \mathrm{~J} / \mathrm{cm}^{2}$.

A performance similar to this behavior would be the benchmark for the "liquid" polymers to allow an application as liquid fuel.

\subsubsection{Ethyl acetate (solvent with carbon)}

The ablation of a liquid target (pure solvent with carbon) yields a different behavior than observed for the solid GAP samples. A cloud of droplets is ejected first, but after several microseconds the liquid surface begins to collapse and jet of liquid is produced (see Figure 3). This behavior can be described as splashing. The splashing is observed for time scales longer than $200 \mu \mathrm{s}$. This splashing is of course detrimental for the application in a laser plasma thruster, because the splashing liquid would contaminate the optics and because energy from the decomposition of the energetic fuel would be lost which would lower the efficiency of the complete system. 

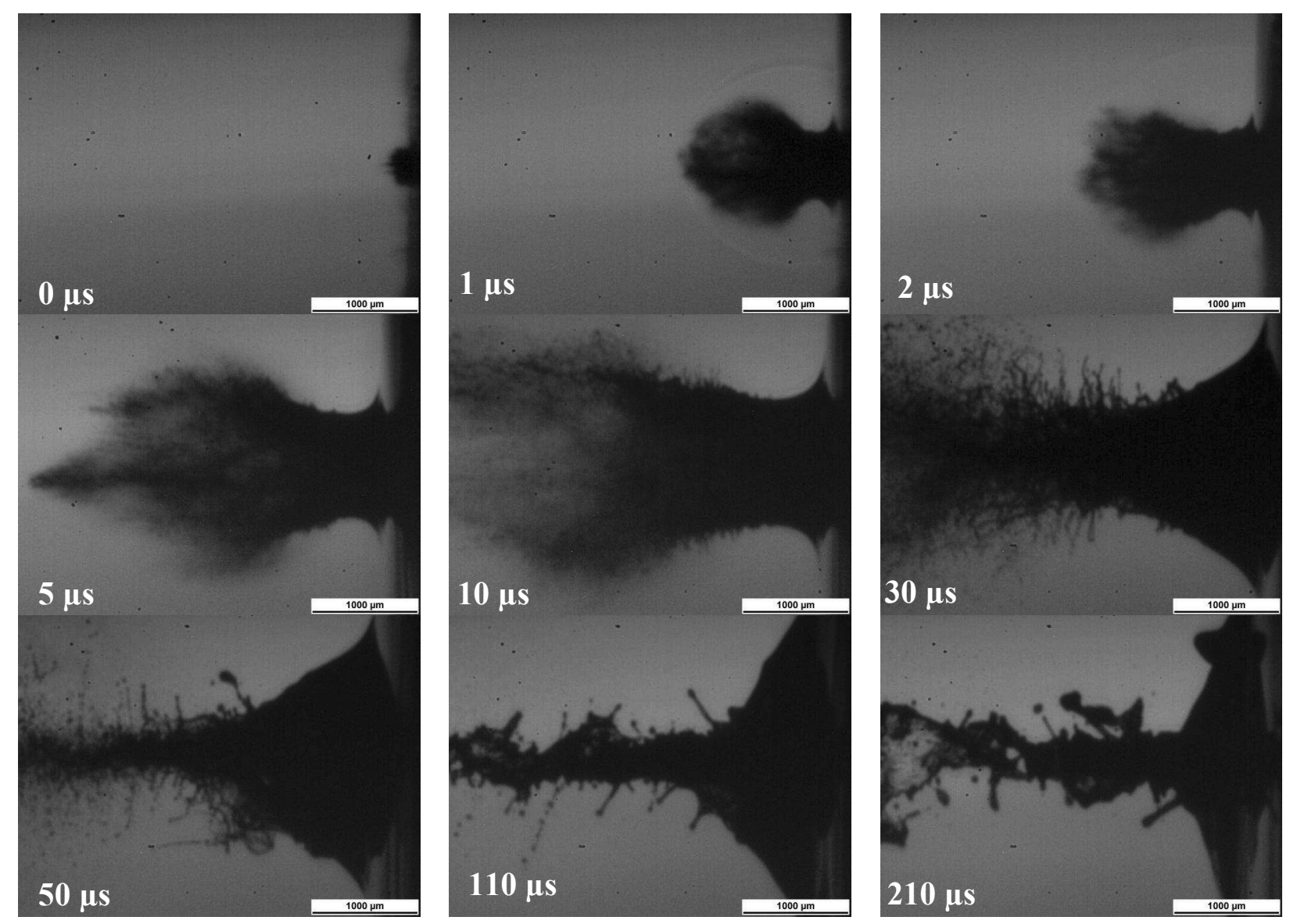

Figure 3: Sequence of pictures taken for ethyl acetate (solvent) with $0.7 \% \mathrm{C}$ particles at $2.8 \mathrm{~J} / \mathrm{cm}^{2}$.

\subsubsection{Non-splashing behavior of GAP solutions}

It is therefore important to identify parameter (laser and material composition) for which splashing can be avoided. Detailed shadowgraphy experiments reveal two possible behaviors during the ablation of liquid GAP solutions. One observed behavior is shown in the Figure 4. 

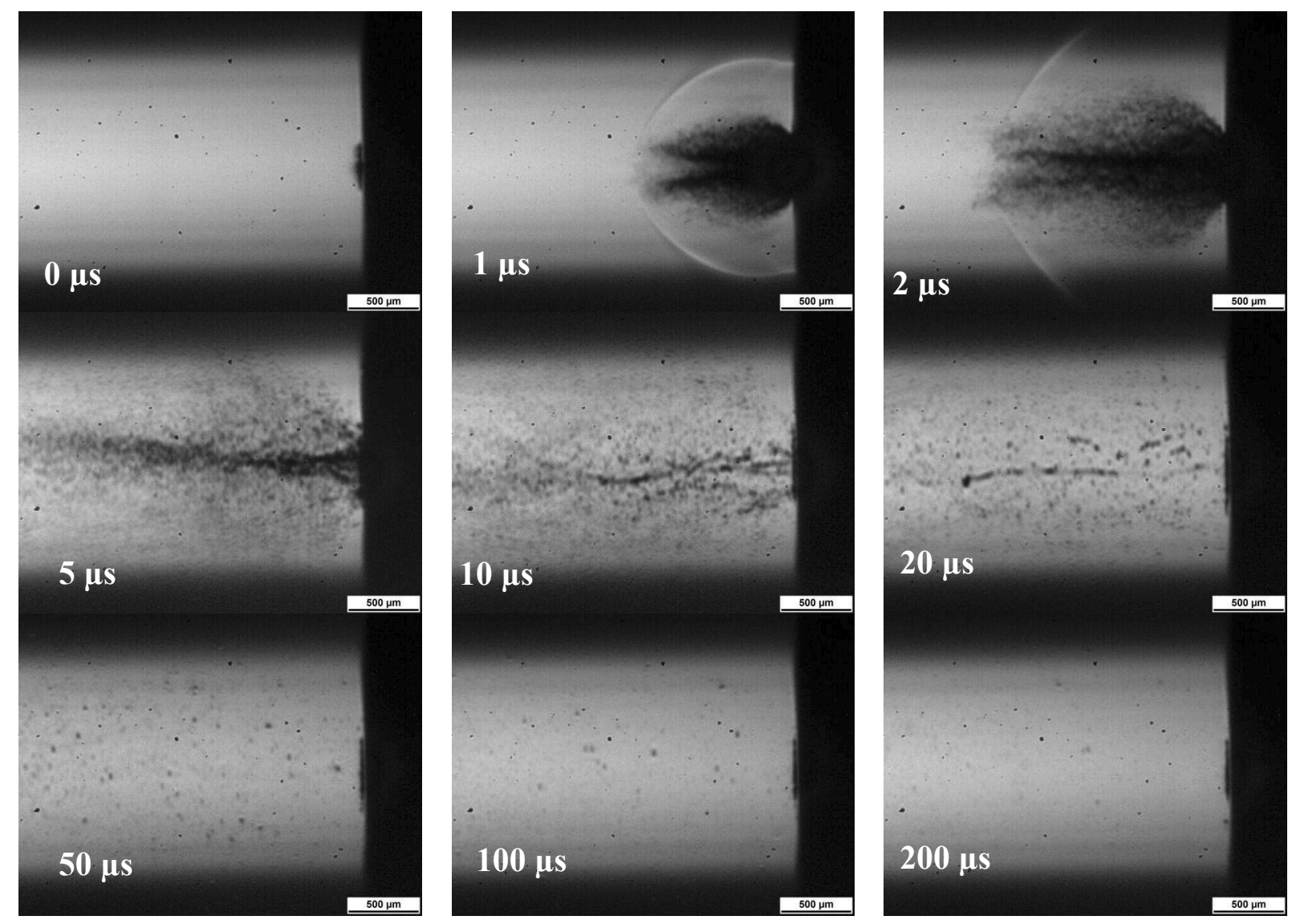

Figure 4: Sequence of pictures taken for the $70 \%$ GAP solution at $2.2 \mathrm{~J} / \mathrm{cm}^{2}$.

Within $5 \mu \mathrm{s}$, the material is ablated and particles are ejected. Remaining particles or droplets are still ejected until 10 to $20 \mu \mathrm{s}$ after the laser pulse. After this time, the ablation process is finished. This process is comparable to the ablation of solid targets, although the solid particles are faster than in the case of solid GAP, i.e. they overtake the shock wave after 1-2 $\mu \mathrm{s}$, which is not observed for solid GAP and the particle/droplet ejection is observed on a longer time scale.

\subsubsection{Splashing behavior of GAP solutions}

The other observed process is the removal of liquid material by splashing. Figure 5 shows the evolution of the sample and area above the sample during splashing. 

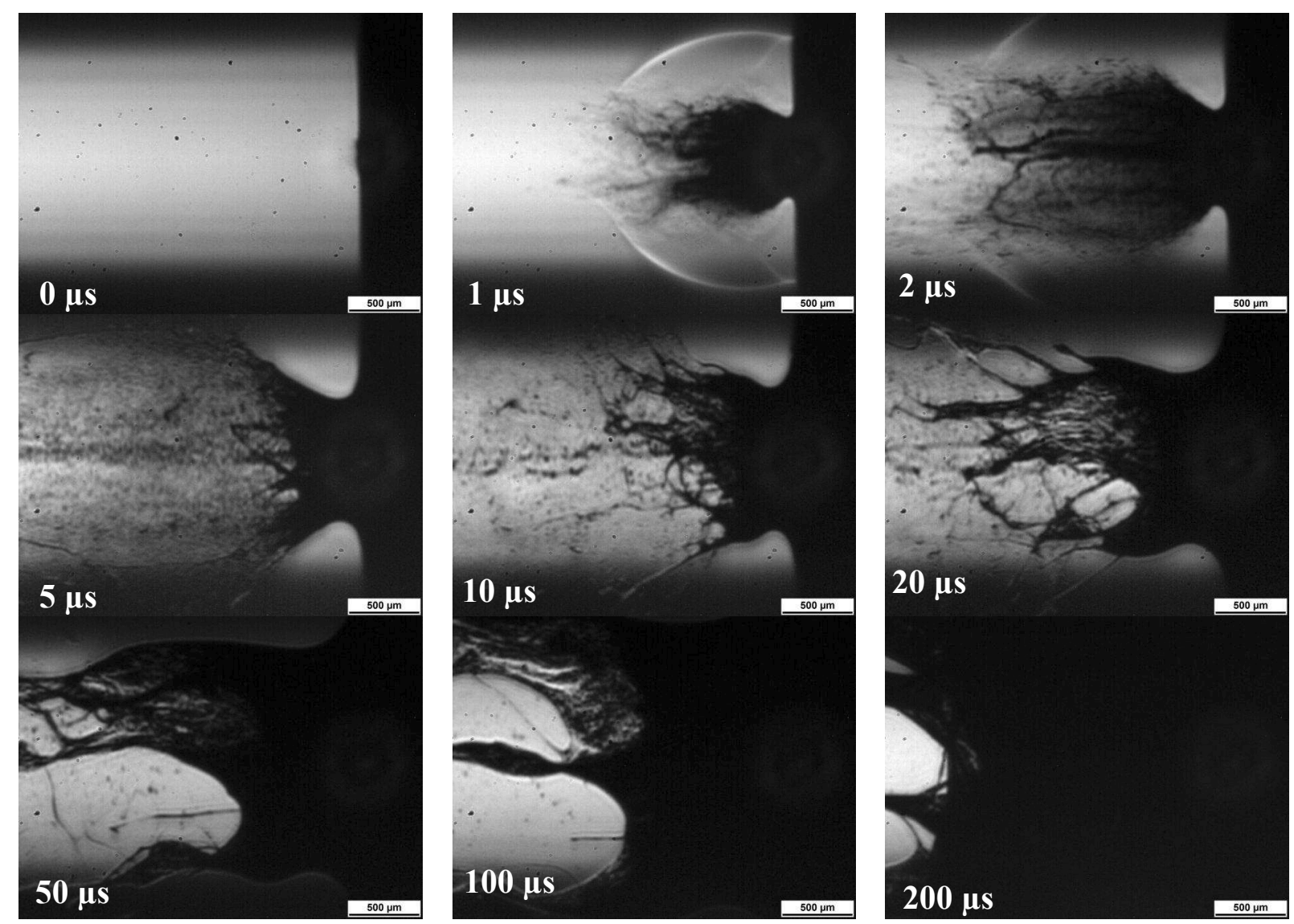

Figure 5: Sequence of pictures taken for the $28 \%$ GAP solution at $4.5 \mathrm{~J} / \mathrm{cm}^{2}$.

The very first steps are similar to the non-splashing ablation. However, two different features can be distinguished: liquid filaments are visible in the plume (which overtake the shockwave at $2 \mu \mathrm{s}$ ), and the base of the plume is connected to the liquid surface by a meniscus. This become particularly clear at $5 \mu \mathrm{s}$, where the liquid surface appears similar to a liquid after impact of a solid particle. The area of the moving liquid is much larger than the original spot size of $300 \mu \mathrm{m}$. After $20 \mu$ s the surface of the liquid seems to expand to several millimeters. After $500 \mu$ s (not shown) the liquid surface is still in an upward movement which is quite different to the behavior of the pure solvent. One possible origin for this difference could be the increased viscosity of the $28 \%$ GAP sample compared to the pure solvent.

\subsubsection{Transition between splashing and non-splashing}

The pictures (Figures 6-10) taken after 9-10 $\mu$ s were chosen to determine which of the two processes is observed for different GAP concentrations and laser fluences.

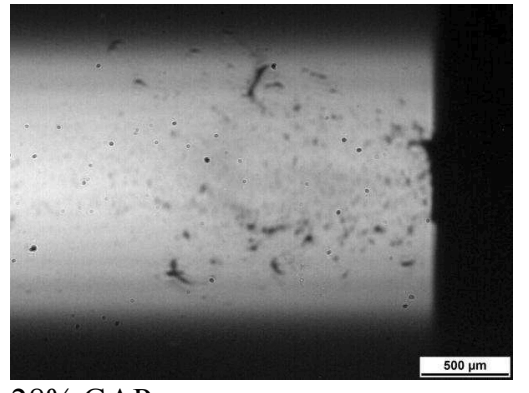

$28 \%$ GAP

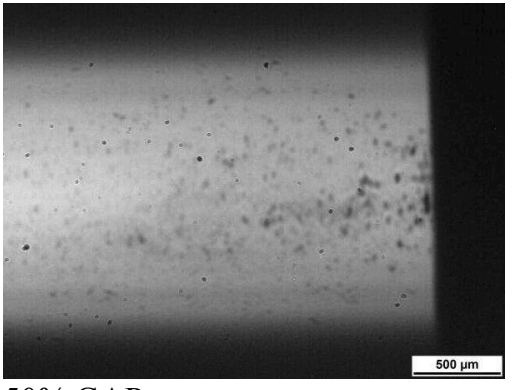

$50 \%$ GAP

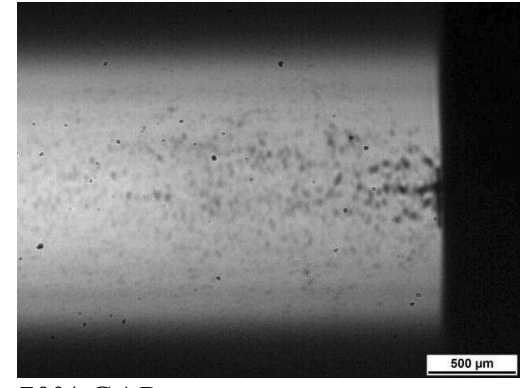

$70 \%$ GAP

Figure 6: Pictures taken after $9 \mu \mathrm{s}$ with $0.55 \mathrm{~J} / \mathrm{cm}^{2}$ for different GAP concentrations. 
At low fluences, all concentrations have the same classical behavior (see Figure 6), and only ejection of small droplets is observed.

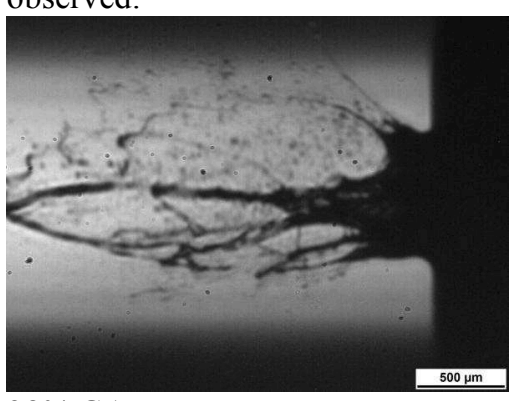

$28 \%$ GAP

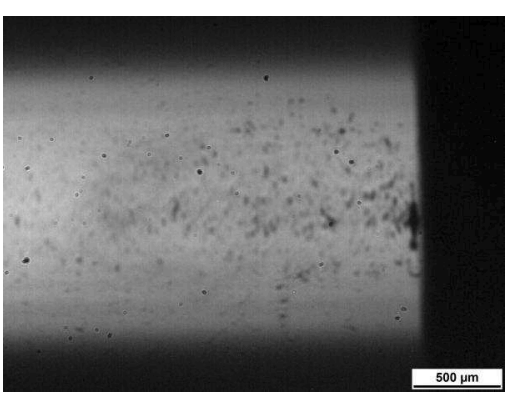

$50 \%$ GAP

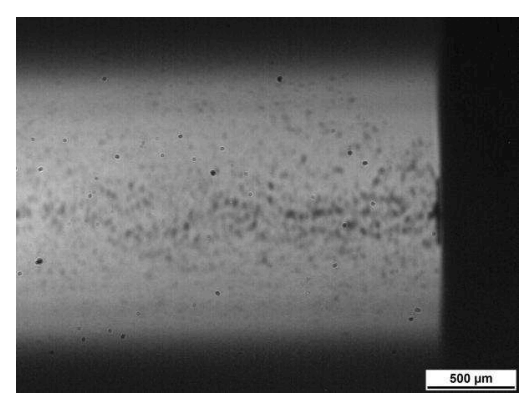

$70 \%$ GAP

Figure 7: Pictures taken after $10 \mu \mathrm{s}$ with $0.8 \mathrm{~J} / \mathrm{cm}^{2}$ for different GAP concentrations.

At higher fluences (see Figure 7), the $28 \%$ GAP, the least viscous solution, shows a splashing behavior but the two other solutions do not reveal this behavior, i.e. only ejection of droplets is observed.

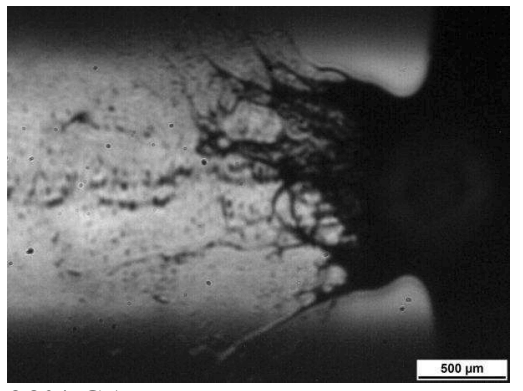

$28 \%$ GAP

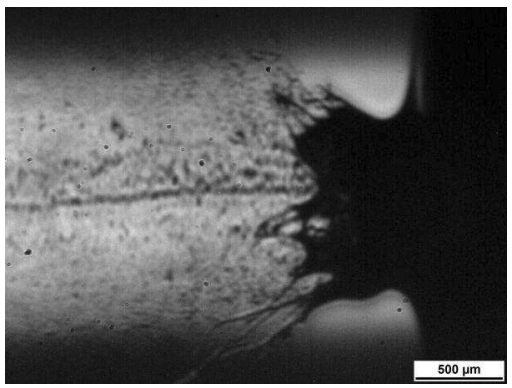

$50 \%$ GAP

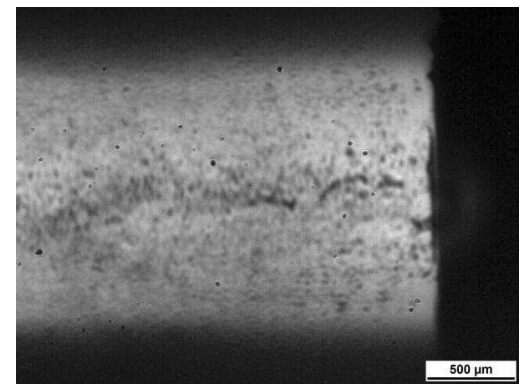

$70 \%$ GAP

Figure 8: Pictures taken after $10 \mu$ s with $4.5 \mathrm{~J} / \mathrm{cm}^{2}$ for different GAP concentrations.

At $4.5 \mathrm{~J} / \mathrm{cm}^{2}$, the $50 \%$ GAP sample is also revealing the splashing behavior while the behavior of the $70 \%$ GAP sample is still similar to a solid (Figure 8).

$\mathrm{n} / \mathrm{a}$
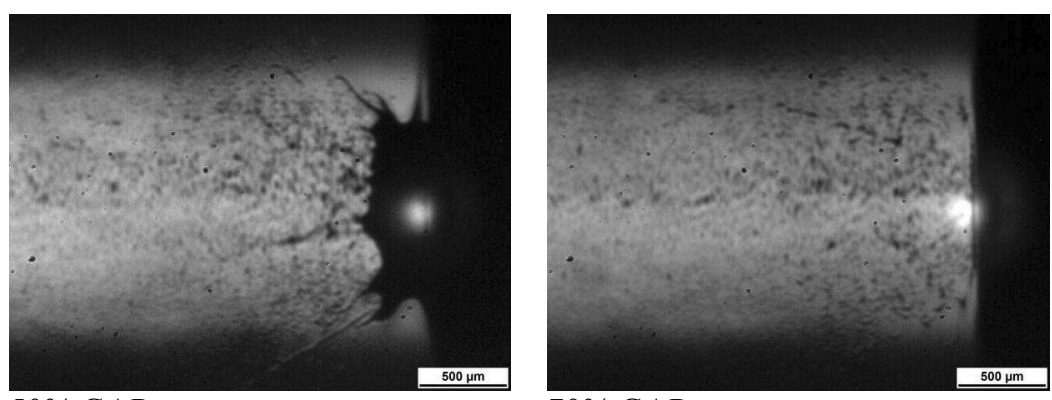

$28 \%$ GAP

$50 \%$ GAP

$70 \%$ GAP

Figure 9: Pictures taken after $10 \mu \mathrm{s}$ with $7.4 \mathrm{~J} / \mathrm{cm}^{2}$ for 2 different GAP concentrations.

The 70\% GAP sample still reveals at even higher fluences $\left(7.4 \mathrm{~J} \mathrm{~cm}^{-2}\right)$ where the plasma onset is already observed (bright spot in the pictures) close to the laser-liquid interaction zone the non-splashing behavior (Figure 9).

For a given concentration of GAP, there is a transition between the solid-like mode and the splashing mode when the fluences are increased. There is also a clear relation between the concentration of the solution and the fluence at which this transition occurs. For solutions with lower GAP concentrations, lower transition fluences are observed. For the $70 \%$ concentration, this transition is not observed in the investigated fluence range, which includes the onset of plasma 
generation. This shows that this concentration behaves similar to solid GAP and is therefore a promising candidate as liquid polymer fuel in a laser plasma thruster.

\subsubsection{Shockwave velocities}

Additional information about the behavior of the different GAP samples can be obtained by the analysis of the shockwaves, which are produced by the ablation. The shock waves can contain information on the energy released during the process. The shockwave evolution versus time was measured for the three GAP solutions. From these data, the velocity at $1 \mu \mathrm{s}$ was calculated and compared to the velocities of the solid GAP and pure solvent. An example of the evolution of the shockwave fronts is given in Figure 10.

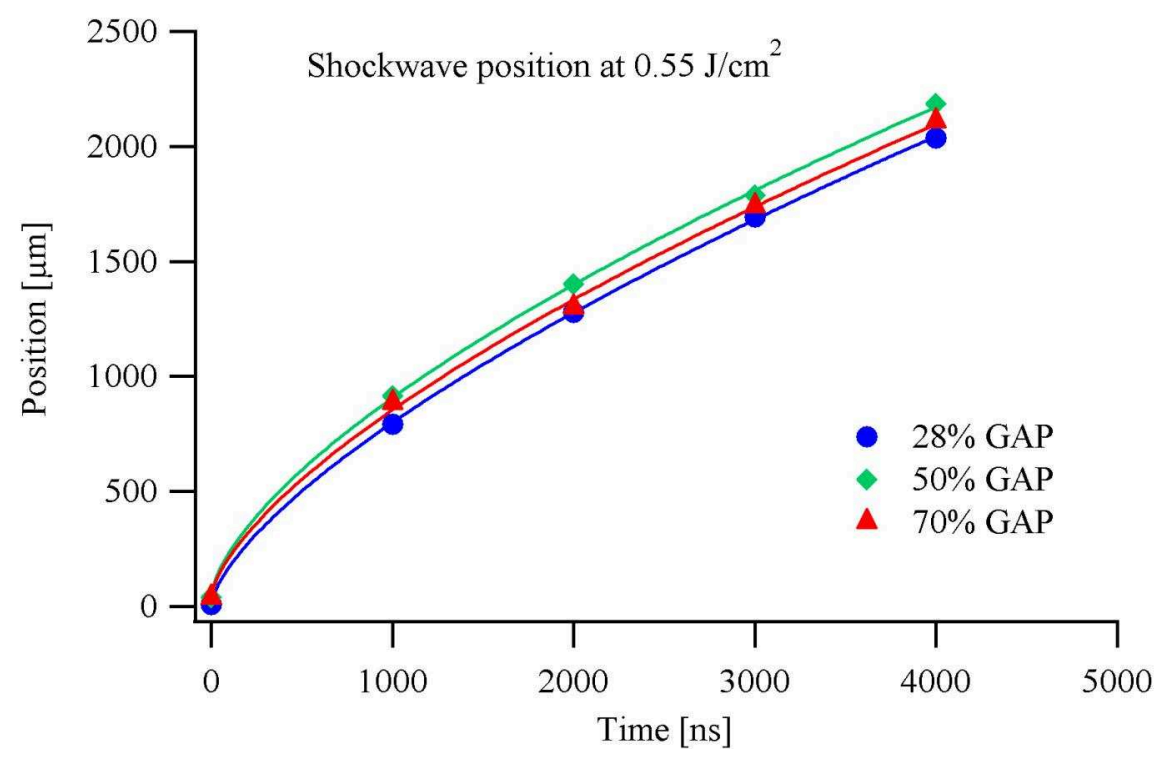

Figure 10: Shockwave front position versus time for different GAP concentration at $0.55 \mathrm{~J} / \mathrm{cm}^{2}$.

The position of the shock wave front was fitted by a power law function,

$$
x(t)=a+b \cdot t^{c}
$$

with $\mathrm{x}=$ the shock wave position, $\mathrm{t}=$ time and $\mathrm{a}, \mathrm{b}, \mathrm{c}$ as fitting parameters.

The velocity at $1 \mu \mathrm{s}$ was obtained by the value of the fit derivative at this time. A summary of all velocities is shown below in Figure 11 for various fluences. 


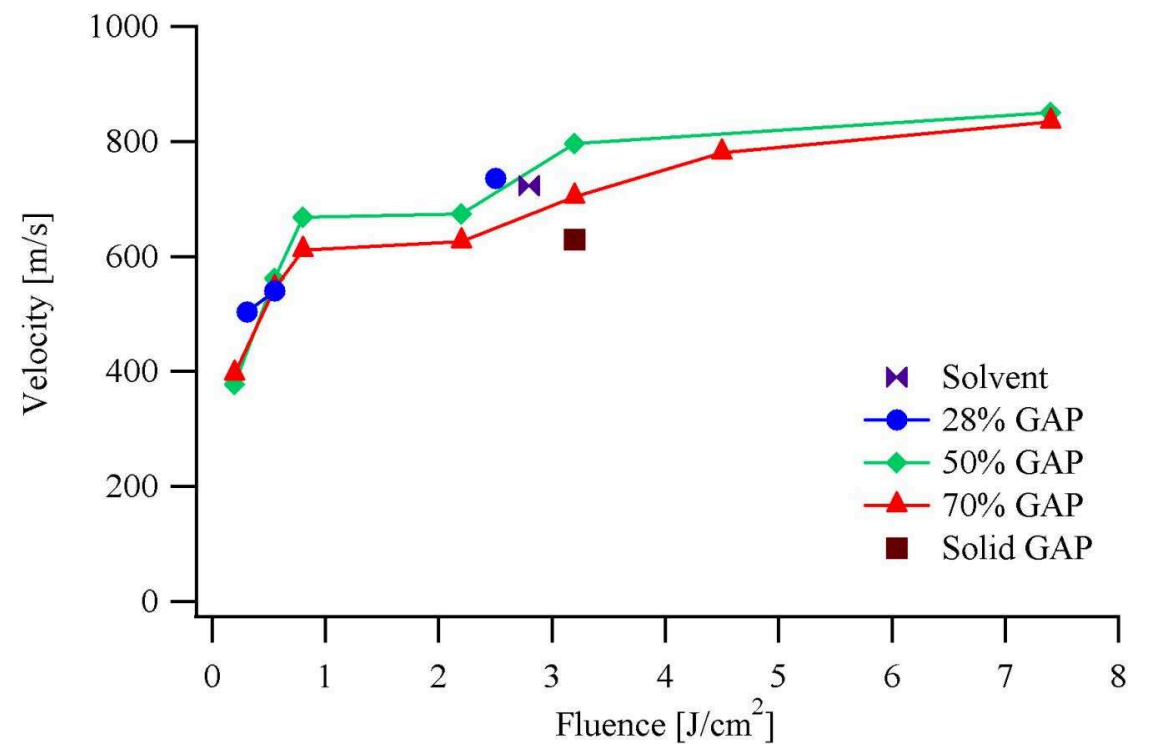

Figure 11: Summary of the shockwave velocities measured at $1 \mu \mathrm{s}$.

The shockwave velocity increases as expected with increasing fluences. This increase is more pronounced for fluences close to the threshold fluence (low fluence range) than for the rest of the investigated fluences. The influence of the GAP concentration is less clear. For a given fluence, all samples have comparable shockwave velocities. However, a slight trend arises from the plot: the higher the GAP content in the target, the slower the shockwave. This result is not really expected, and a possible correlation of the splashing transition with the velocities of the shock wave front seems to be impossible. One possible explanation for this effect may be related to the low boiling point of ethyl acetate $(\sim 350 \mathrm{~K})$. The solvent is readily vaporized and therefore more energy can be released by the shock wave, yielding faster shock waves for samples with higher solvent content.

These data suggest also clearly that GAP solution with a concentration of $\geq 70 \%$ of GAP can be applied as fuel in micro laser plasma thrusters, and that a thrust similar to solid samples may be obtained.

\subsubsection{Viscosity of the GAP solutions and preliminary thrust measurements}

The most probable explanation for the transition from splashing to non-splashing behavior with increasing GAP concentration is the increasing viscosity of the GAP solutions with increasing GAP content. The viscosity has therefore been measured (see Table 2) to quantify the observed differences between the different GAP concentrations.

Table 2: Viscosity of different solutions of GAP

\begin{tabular}{cc}
\hline Solution & Dynamic viscosity $[\mathrm{Pa} \mathrm{s}]$ \\
\hline $28 \%$ GAP & 0.012 \\
$50 \%$ GAP & 0.46 \\
$70 \%$ GAP & 14 \\
\hline
\end{tabular}

The three solutions have very different rheological properties, as the dynamic viscosity increases very fast for GAP concentrations $>50 \%$. It appears that the required viscosity to apply liquid GAP solution as fuel for micro laser plasma thruster is in the range of 10-15 $\mathrm{Pa}$ s which should still be possible to deliver by a pumping system.

First preliminary tests with $70 \%$ GAP and an added IR absorber show that this concentration behaves, as expected from our results, similar to solid samples, yielding a specific impulse of $680 \mathrm{~s}$ which is much higher then the reported specific impulse for doped water of $19.7 \mathrm{~s}^{[13]}$ and even higher than the value for the solid GAP sample of $\sim 250 \mathrm{~s}$. 


\section{CONCLUSIONS}

The application of energetic polymers has resulted in an increased thrust in micro laser plasma thrusters compared to standard polymers. In this study we tested a novel concept for micro laser plasma thrusters, i.e. the application of liquid polymeric fuels, by using polymer solutions of the energetic materials with different viscosity, because non-viscous liquids yield a quite low specific impulse due to pronounced splashing of the liquid. This splashing lowers drastically the efficiency and specific impulse of the liquid fuel. Shadowgraphy experiments have been performed to analyze the ablation behavior, i.e. whether splashing can be detected. The shadowgraphy results show clearly that there is an onset of splashing for a given GAP concentrations (below 70\% of GAP) with increasing laser fluence, and that this onset is increases with increasing GAP concentrations. For a GAP concentration of $70 \%$ no-splashing is observed for fluences up to the plasma onset. This shows that for higher viscosity solutions ablation without splashing is possible, indicating that liquids are applicable as fuels in laser plasma thrusters. First thrust measurements on a viscous polymer solution confirmed this by yielding a specific impulse similar to a solid material.

\section{ACKNOWLEDGEMENTS}

This effort has been sponsored by the Air Force Office of Scientific Research, Air Force Material Command, USAF, under grant number FA8655-03-1-3058 and EOARD contract SPC 074015.

The author thanks Dr. F. Winnefeld for his support and expertise with the rheological measurements at Empa's Concrete/Construction chemistry Laboratory and S. Heiroth for the preparation of the samples.

\section{REFERENCES}

${ }^{[1]}$ C. Phipps and J. Luke, AIAA Journal 40, 310 (2002).

${ }^{[2]}$ C. Phipps, J. Luke, T. Lippert, M. Hauer, and A. Wokaun, J. Propul. Power 20, 1000 (2004).

${ }^{[3]}$ C. R. Phipps, J. R. Luke, T. Lippert, M. Hauer, A. Wokaun, Micropropulsion Using a Laser Ablation Jet, J. Prop. Power 20,1000 (2004).

${ }^{[4]}$ C. R. Phipps, J. R. Luke, T. Lippert, Laser Ablation of Organic Coatings as a basis for Micropropulsion, Thin Solid Films, 453454, 573 (2004).

${ }^{[5]}$ C. R. Phipps, J. R. Luke, G. G. McDuff, T. Lippert, Laser-driven micro-rocket, Appl. Phys. A 77, 193 (2003).

${ }^{[6]}$ C. R. Phipps, J. R. Luke, G. G. McDuff, T. Lippert, Laser ablation powered mini-thruster, SPIE Proceedings Vol. 4760,833 (2002).

${ }^{[7]}$ T. Lippert, M. Hauer, C. R. Phipps, A. Wokaun, Polymers designed for laser applications: fundamentals and applications, invited paper, SPIE Proceedings Vol. 4760, 63 (2002).

${ }^{[8]}$ T. Lippert, M. Hauer, C. Phipps, A. Wokaun, Fundamentals and applications of polymers designed for laser ablation, Appl. Phys. A 77, 259 (2003).

${ }^{[9]}$ L. Urech, T. Lippert, C. R. Phipps, A. Wokaun, Polymers as fuel for laser plasma thrusters: A correlation of thrust with material and plasma properties by mass spectrometry, Proc. SPIE 6261, 6261114-1 (2006).

${ }^{[10]}$ L. Urech, T. Lippert, Designed polymers for ablation, invited chapter in Laser Ablation and its Applications, Springer Series in Optical Sciences, Vol. 129, Ed. C. Phipps, Springer 2007, p. 281-297.

${ }^{[11]}$ L. Urech, M. Hauer, T. Lippert, C. Phipps, E. Schmid, A. Wokaun, and I. Wysong, Proc. SPIE 5448, 52 (2004).

${ }^{[12]}$ L. Urech, T. Lippert, C. R. Phipps, A. Wokaun, Polymers as fuel for laser-based microthrusters: an investigation of thrust, material, plasma and shockwave properties, Appl. Surf. Sci. 253, 6409 (2007).

${ }^{[13]}$ T. Yabe, C. Phipps, M. Yamaguchi, R. Nakagawa, K. Aoki, H. Mine, Y. Ogata, C. Baasandash, M. Nakagawa, E. Fujiwara, K. Yoshida, A. Nishiguchi, Appl. Phys. Lett. 80, 4318 (2002)

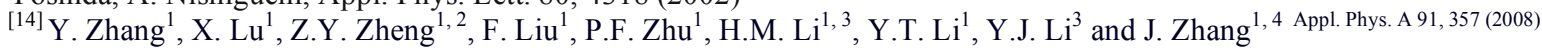

